Bulletin of Mathematical Biology (2002) 64, 623-641

doi:10.1006/bulm.2001.0271

Available online at http://www.idealibrary.com on IDE $A \mathbf{L}^{\circledR}$

\title{
Modelling Blood Flow Regulation by Nitric Oxide in Psoriatic Plaques
}

\author{
JONATHAN A. SHERRATT \\ Centre for Theoretical Modelling in Medicine, \\ Department of Mathematics, Heriot-Watt University, \\ Edinburgh EH14 4AS, \\ U.K. \\ E-mail: jas@ma.hw.ac.uk
}

\section{RICHARD WELLER}

Department of Surgery, UPMC,

E 1557 Biomedical Science Tower,

200 Lothrop Street,

Pittsburgh, PA 15261,

U.S.A.

E-mail: wellerr@msx.upmc.edu

\section{NICHOLAS J. SAVILL}

Centre for Theoretical Modelling in Medicine,

Department of Mathematics, Heriot-Watt University, Edinburgh EH14 4AS,

U.K.

E-mail: njs@ma.hw.ac.uk

Psoriasis is a common skin disease, with a clinical appearance of red, scaly lesions, known as plaques. Recent experimental research has shown that the ubiquitous cellsignalling molecule nitric oxide (NO) is actively synthesized within these plaques by the iNOS enzyme. In contrast, NO production from normal, healthy skin is a byproduct of the reduction of nitrite in sweat. Measurement of NO release rates at the skin surface are 100 times greater from psoriatic lesions than normal skin. We propose a mathematical model for the dynamics of $\mathrm{NO}$ within psoriatic plaques, that incorporates diffusion, production in the basal epidermis, decay within the plaque, and active scavenging by red blood cell haemoglobin; this last effect introduces a key nonlinearity into the model. We present numerical simulations of the model in two space dimensions, and then describe an approximation that reduces the model to two coupled ordinary differential equations. This reduced system can be solved exactly, giving an approximation for the NO release rate as an explicit function of model parameters. We use this approximation to explain some recent, surprising experimental results.

(c) 2002 Society for Mathematical Biology. Published by Elsevier Science Ltd. All rights reserved. 


\section{INTRODUCTION}

Psoriasis is a common skin disease, affecting about $2 \%$ of Caucasian populations. In the most common forms, raised, inflamed (red) lesions, known as plaques, develop on the skin surface, covered by a silvery, white scale. The outer layer of the skin (epidermis) is significantly thickened, with increased cell proliferation in the basal layer and incomplete cell differentiation. The epidermis is also infiltrated by large numbers of white blood cells, which cluster in small abscesses. In the lower layer of skin (dermis) the capillary loops are tortuous and dilated leading to increased blood flow and skin redness. The interface between the dermis and epidermis also differs from normal skin. The thickened epidermis protrudes into the dermis forming fingers called rete pegs and dermal papillae containing the capillary loops.

Despite this detailed understanding of the psoriatic pathologies, relatively little is known about the underlying molecular events that cause the disease, and this is the focus of current psoriasis research. One recent line of investigation concerns the signalling molecule nitric oxide (NO). Long known as an environmental pollutant, it was shown in the late 1980s to be biologically active, and a huge volume of subsequent research has established $\mathrm{NO}$ as an important regulator in most tissues of the body (for review see Moncada, 1999).

In particular, NO is produced at high rates within psoriatic plaques. Due to its small size and solubility in phospholipid membranes, NO has a high diffusion coefficient in tissue. Thus it can easily be detected at the skin surface. Weller et al. (1996) designed an experiment to do just that; they placed a sealed nitrogenfilled vessel over the psoriatic plaque, allowed NO to diffuse into the vessel from the skin for $20 \mathrm{~min}$, then aspirated the accumulated NO into a chemiluminescence analyser. They showed that in healthy human skin, NO is released at relatively low levels, due mainly to chemical reactions occurring within the sweat layer that covers the skin. By contrast, NO is released from psoriatic plaques at a rate about 100 times greater (Orem et al., 1997; Weller and Ormerod, 1997; Ormerod et al., 1998).

These results suggest that NO might play a causal role in psoriasis, a possibility which would have exciting potential therapeutic implications, and for this reason, $\mathrm{NO}$ activity in psoriasis is an ongoing research area. In this paper, we use a mathematical model to study the dynamics of $\mathrm{NO}$ within a psoriatic plaque, investigating the NO distribution, and how the release rate at the skin surface depends on model parameters. More specifically, we attempt to explain recent results of Weller et al. (manuscript in preparation), who investigated the relationship between the redness (erythema) of psoriatic plaques, and NO release rates from their surface; their data is illustrated in Fig. 1. NO is a potent dilator of blood vessels: this was the first context in which the biological activity of NO was recognized (Ignarro et al., 1987). Therefore, one expects intuitively that high NO release rates will correlate with high plaque erythema. However, Weller et al. found that while there was a positive 


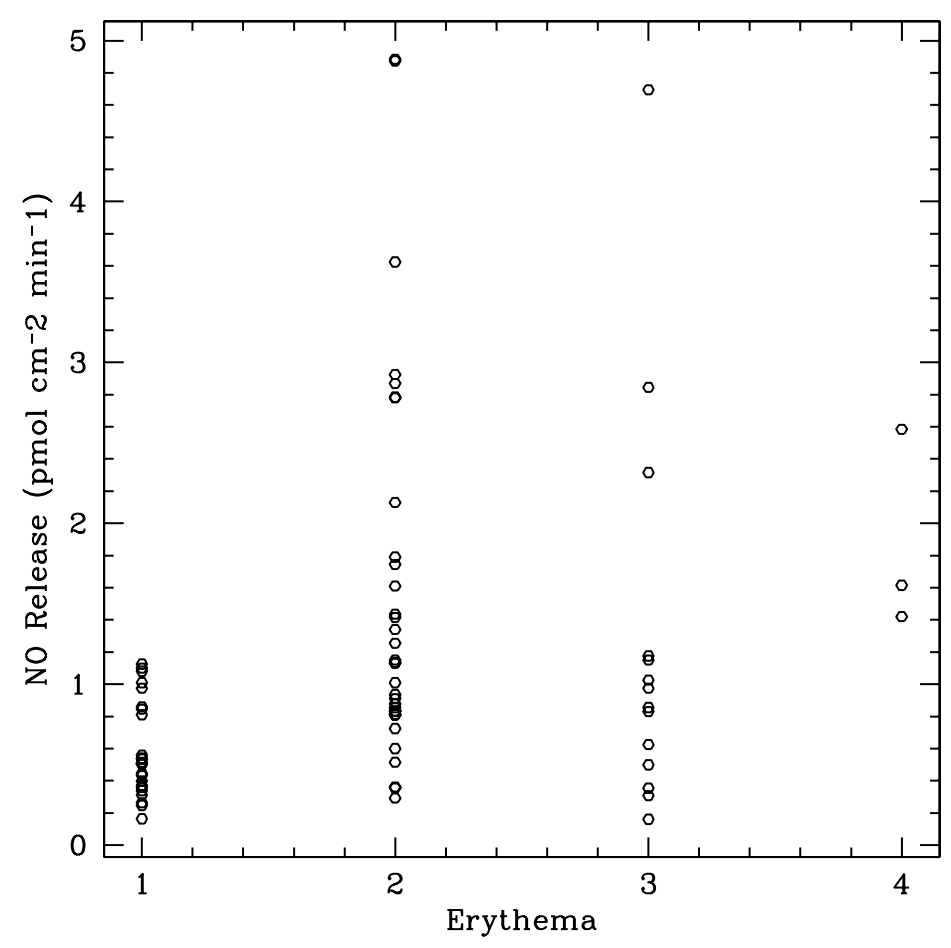

Figure 1. Experimental data showing the relationship between the erythema of psoriatic plaques and NO release rates from their surface. Full details of these experiments will be published separately (Weller et al., in preparation). Briefly, erythema was scored by a single observer (RW) on the scale 1 (mild), 2 (moderate), 3 (severe) and 4 (very severe). NO released from the plaque was collected in a nitrogen filled container over a $20 \mathrm{~min}$ period, and the concentration was then measured using a chemiluminescence meter.

correlation between the two measurements, NO release was actually highest in plaques with moderate erythema, with lower average NO release in plaques with either high or low erythema. One of our objectives is to explain this observation using mathematical modelling.

In Section 2 we develop a mathematical model for NO dynamics in psoriasis. The model equation can be solved numerically, but not analytically in its full form, and in Section 3 we discuss an approximate model for the equilibrium dynamics of NO. The approximate model is not derived systematically, but rather via a series of ad hoc, but biologically reasonable, approximation steps. The approximate model can be solved exactly, and this is done in Section 4. Finally, in Section 5, we discuss the implications of our results.

\section{Mathematical Model}

We develop a mathematical model for NO dynamics within a psoriatic plaque, consisting of a single reaction-diffusion equation for the NO concentration $n(\underline{r}, t)$. 
The kinetics of NO within a plaque involve three main processes. Firstly, synthesis of NO, which occurs in cells in the basal layer of the epidermis; this has been established by staining tissue samples for the enzyme iNOS that causes the production (Kolb-Bachofen et al., 1994; Ormerod et al., 1998). This is not the only source of NO within a psoriatic plaque: NO is also produced in the dermal papillae and in some cases in the suprabasal epidermis (Bruch-Gerharz et al., 1996). However, we assume that the NO production in the basal epidermis is dominant, and occurs at a constant rate, $a$. There is no experimentally determined value for this parameter; hence, we will estimate it by matching the observed average release rate of NO from psoriatic plaques with that predicted by the model. Secondly, NO decays within tissues, due mainly to reaction with superoxide $\mathrm{O}_{2}^{-}$. This gives a half life of about $4 \mathrm{~s}$ (Lancaster, 1994), approximately the same in different parts of the plaque; we model this with the term $-\lambda n$, with the constant $\lambda=0.17 \mathrm{~s}^{-1}$.

Thirdly, NO is actively removed by red blood cell haemoglobin within the dermal capillaries. This occurs in addition to the $-\lambda n$ decay term. Crucially, though, this is a nonlinear process: NO dilates blood vessels causing increased blood flow (and increased redness) and hence greater NO scavenging by haemoglobin. Thus, the removal rate of NO within the dermal papillae has the form $-v n[1+g(n)]$, where $g(n)$ is the increase in the cross-sectional area of blood vessels in response to NO. In vitro experiments on blood vessels, where NO levels can be externally controlled, show that an appropriate functional form is $g(n)=g_{0} n /\left(g_{1}+n\right)$, where $g_{1} \approx 20 \mathrm{nM}(\mathrm{Ku}, 1996)$ and $g_{0}$ is the maximal change in cross-sectional area of the capillaries. The value of $g_{0}$ is unknown and will vary between individuals, but estimates suggest that the blood flow in psoriatic plaques is two to five times greater than in normal skin (Klemp and Staberg, 1983; Krogstad et al., 1995). Assuming Poiseuille flow in the capillaries implies that $1+g(n)$ varies between about 1.4 and $2.2(\sqrt{2}-\sqrt{5})$. Like the production rate $a, g_{0}$ can be found by matching observation with simulation. The parameter $v$ is the product of haemoglobin concentration in the blood (typically $2 \mathrm{mM}$; Thibodeau and Patton, 1997), the proportion of the dermal papillae composed of blood vessels (about 10\%; Auer et al., 1994), and the rate constant of NO binding to haemoglobin in flowing blood $\left(5 \times 10^{4} \mathrm{M}^{-1} \mathrm{~s}^{-1}\right.$; Liao et al., 1999); this gives $v=10 \mathrm{~s}^{-1}$.

Putting these various terms together gives the following equation for NO dynamics in a plaque:

$$
\frac{\partial n}{\partial t}=D \nabla^{2} n+ \begin{cases}-\lambda n & \text { in suprabasal epidermis } \\ -\lambda n+a & \text { in basal epidermis } \\ -\lambda n-v n\left[1+g_{0} /\left(g_{1}+n\right)\right] & \text { in dermis. }\end{cases}
$$

Here $D$ is the diffusion coefficient of NO in tissue, which has been measured experimentally as $3300 \mu \mathrm{m}^{2} \mathrm{~s}^{-1}$ (Malinski et al., 1993). We have solved these equations numerically in the two-dimensional (2D) spatial domain illustrated in Fig. 2, which corresponds roughly to the geometry of a psoriatic plaque. We denote by $Y, h$ and 


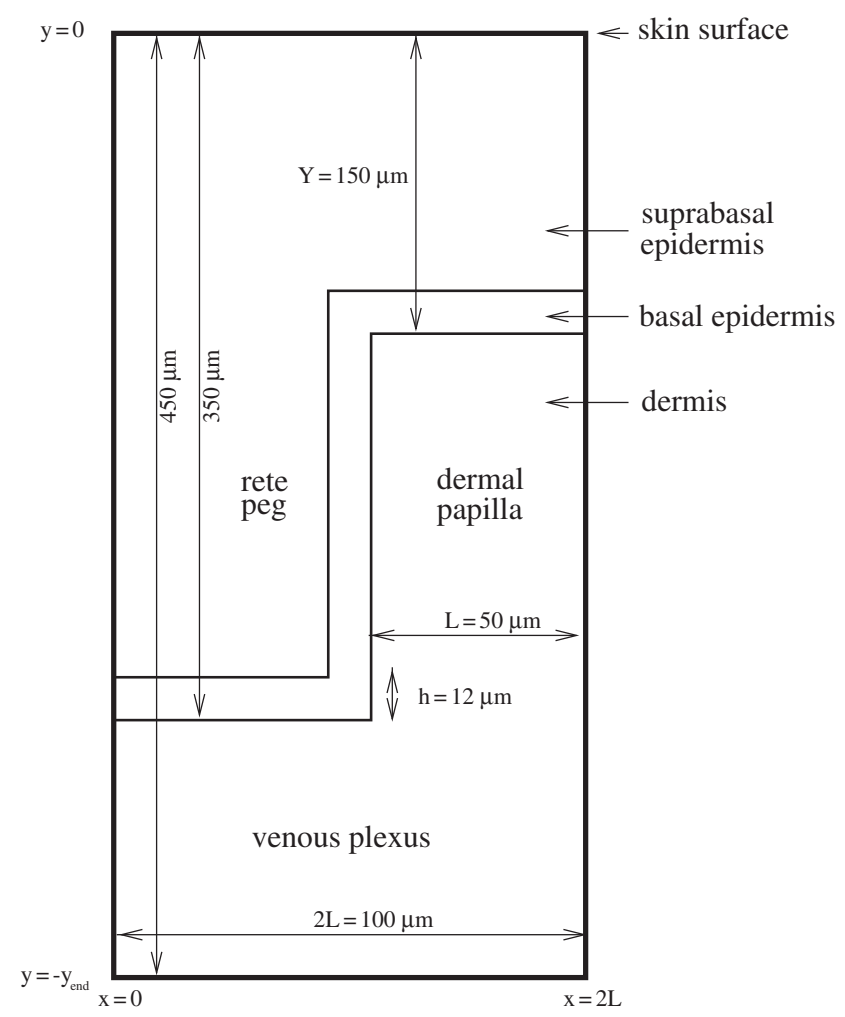

Figure 2. A schematic illustration of the spatial domain on which we solve the full model (1).

$L$ the thickness of epidermis above a dermal papilla, the thickness of the basal epidermis, and the half-width of a rete ridge/dermal papilla, respectively. Typical values for a psoriatic plaque are $Y=150 \mu \mathrm{m}, h=12 \mu \mathrm{m}$ and $L=50 \mu \mathrm{m}$. Symmetry boundary conditions apply at $x=0$ and $x=2 L$, which are the middle of an epidermal ridge and a dermal papilla, respectively. At $y=0$, which is the skin surface, we take $n=0$. This is appropriate since the diffusion coefficient of NO in air is about 1000 times greater than in tissue, so that the NO released into the air diffuses away rapidly. Note that it is the NO flux at the skin surface, rather than concentration, that is measured experimentally. The boundary condition at $y=-y_{\text {end }}$ is included for computational necessity; we take $n_{y}=0$, but it is not significant provided that $y_{\text {end }}$ is sufficiently large.

The solution of (1) rapidly evolves to an equilibrium, independent of initial data. A typical example of this equilibrium is illustrated in Fig. 3; the NO concentration is highest in the basal layer of the epidermis, where it is produced, moderate in the suprabasal epidermis, and lowest in the dermis, where it is actively removed into the blood stream. We have good estimates for all parameters except $a$ and $g_{0}$. These two parameters can be determined by comparing the results of model 


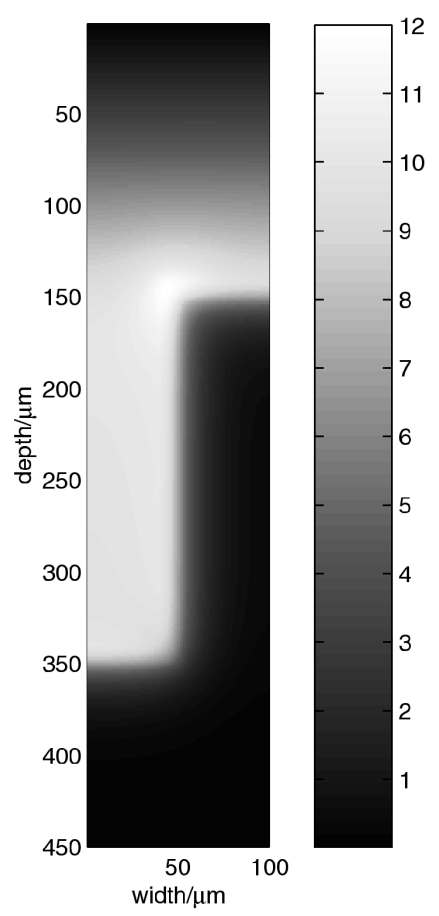

Figure 3. A typical solution of the full model (1), showing the equilibrium distribution of NO within a psoriatic plaque. This equilibrium is reached very rapidly (typically within a few seconds); the scale bar gives the NO concentration in $\mathrm{nM}$. The skin surface $y=0$ is at the top of the picture. The model equation (1) was solved numerically using an alternating direction implicit Crank-Nicolson scheme. Parameter values are $D=3300 \mu \mathrm{m}^{2} \mathrm{~s}^{-1}$, $\lambda=0.17 \mathrm{~s}^{-1}, a=170 \mathrm{nM} \mathrm{s}^{-1}, v=10 \mathrm{~s}^{-1}, g_{0}=9$ (dimensionless), $g_{1}=20 \mathrm{nM}$, $Y=150 \mu \mathrm{m}, h=12 \mu \mathrm{m}, L=50 \mu \mathrm{m}$.

solutions with two experimental measurements on psoriatic plaques: the rate of NO release from the plaque surface (mean $1.2 \mathrm{pmol} \mathrm{cm}^{-2} \mathrm{~min}^{-1}$; calculated from the data in Fig. 1) and the increase in blood flow in the plaque compared to normal skin (factor of 1.8; Klemp and Staberg, 1983; Krogstad et al., 1995). This data relates directly to model solutions: the model prediction of NO flux at the skin surface is the average over $x$ of $-D \partial n /\left.\partial y\right|_{y=0}$, and the prediction of erythema is the average over the dermal papilla of $1+g(n)$. Comparison of these predictions with the data implies mean values of $a=170 \mathrm{nM} \mathrm{s}^{-1}$ and $g_{0}=9$ (dimensionless); however, these parameters will vary between individuals.

There is wide variation in the NO flux and erythema between patients. Determining how these two aspects of the disease depend on model parameters using the full model (1) is very cumbersome-since analytical solution is not possible, it would require large numbers of numerical simulations in two space dimensions. Therefore, a simpler, analytically solvable model would be preferred. In the next section we develop such a model. 


\section{Approximate Model}

One of our objectives in this paper is to study the variation of NO release rates from psoriatic plaques with plaque erythema. This can be done most effectively by obtaining analytical formulae for the flux and erythema, as a function of model parameters. In this section, we discuss an approximate version of (1) at equilibrium, in which such analytical solution is possible. Having developed this model we will go on, in Section 4, to derive expressions for NO flux and erythema for this approximate model, which will enable us to investigate their relationship.

Our approximate model is based on an ad hoc reduction of the full system, which replaces (1) at equilibrium with two coupled ordinary differential equations. The approximate model therefore has no formal relationship with the full model, but does provide an effective qualitative and semi-quantitative representation of the behaviour of the full model, with sufficient simplicity to be solved exactly. The approximation involves three basic steps.

Step 1. We replace the function $g(n)=g_{0} n /\left(g_{1}+n\right)$ with the linear expression $g_{0} n / g_{1}$. This is reasonable since the NO concentration $n$ is relatively low in the dermis, compared to $g_{1}=20 \mathrm{nM}$.

Step 2. The most fundamental step of our approximation is that we consider the epidermal peg and dermal papilla separately from the remainder of the epidermis (illustrated in Fig. 4). In the lower layer $\mathcal{L}$ (epidermal peg/dermal papilla), we neglect variation perpendicular to the skin surface, giving an ODE for $n$ as a function of $x$ only. As in the full model (1), this ODE has different kinetics in the three regions: the suprabasal epidermis $\left(\mathcal{L}_{1}\right)$, the basal epidermis $\left(\mathcal{L}_{2}\right)$ and the dermis $\left(\mathcal{L}_{3}\right)$. In the upper layer $\mathcal{U}$ (remainder of the epidermis), we similarly neglect variation parallel to the skin surface, giving an ODE for $n$ as a function of $y$. Again, the kinetics are different in the basal $\left(\mathcal{U}_{1}\right)$ and suprabasal $\left(\mathcal{U}_{2}\right)$ regions; in $\mathcal{U}_{1}$, we take the NO production rate to be $a / 2$, representing an average of the basal layer above the dermal papilla, and the suprabasal epidermis above the rete peg (see Fig. 2). We link the solutions in the two layers $\mathcal{L}$ and $\mathcal{U}$ by requiring that the value of $n$ at $y=-Y$ in $\mathcal{U}$ is equal to the average value of $n$ over the regions $\mathcal{L}_{1}, \mathcal{L}_{2}$ and $\mathcal{L}_{3}$.

Step 3. Finally, we remove the boundary condition at $x=2 L$ in region $\mathcal{L}_{3}$, which greatly complicates the analytical solution. Instead we solve the equation that applies in $\mathcal{L}_{3}$ on the domain $L<x<\infty$, with boundary condition $n \rightarrow 0$ as $x \rightarrow \infty$.

For notational ease, we write $\alpha=\sqrt{(\lambda+v) / D}$ and $\beta=g_{0} v /\left(g_{1} D\right)$; then our approximate model is given by

$$
\begin{aligned}
D n_{x x}-\lambda n=0 & \text { in } \mathcal{L}_{1}, \text { that is } 0<x<L-h \\
D n_{x x}-\lambda n+a=0 & \text { in } \mathcal{L}_{2}, \text { that is } L-h<x<L
\end{aligned}
$$




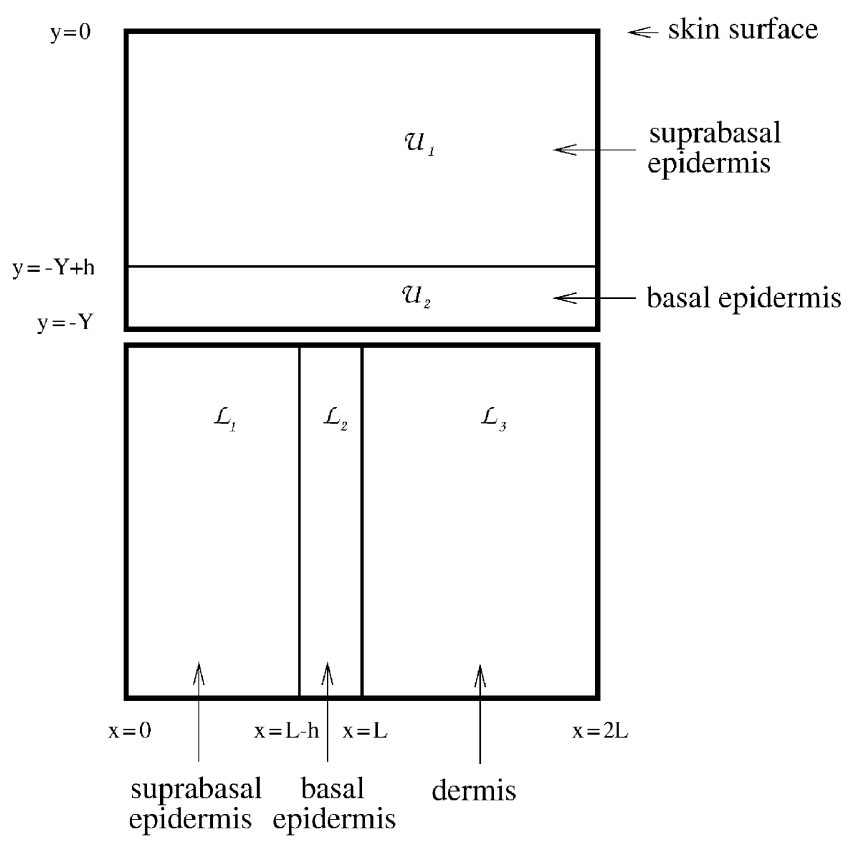

Figure 4. A schematic illustration of our approximation to the full model, which should be compared with Fig. 2. We divide the domain into two layers: the lower layer $\mathcal{L}$, consisting of the dermal papilla and epidermal peg; and the upper layer $\mathcal{U}$, consisting of the remainder of the epidermis. In $\mathcal{L}$, we solve for $n$ as a function of $x$ only, while in region $\mathcal{U}, n$ is a function of $y$. The equations of the approximate model are (2)-(6).

$$
\begin{aligned}
& n_{x x}-\alpha^{2} n-\beta n^{2}=0 \text { in } \mathcal{L}_{3}, \text { that is } L<x<2 L \\
& \text { (but solved on } x>L \text { subject to } \\
&n \rightarrow 0 \text { as } x \rightarrow \infty) \\
& D n_{y y}-\lambda n=0 \quad \begin{array}{ll}
\text { in } \mathcal{U}_{1}, & \text { that is }-Y+h<y<0 \\
D n_{y y}-\lambda n+a / 2=0 & \text { in } \mathcal{U}_{2}, \text { that is }-Y<y<-Y+h .
\end{array}
\end{aligned}
$$

As in (1), we take $n=0$ at $y=0$, the skin surface, and $n_{x}=0$ at $x=0$, the centre of the epidermal peg. The solution must satisfy continuity and smoothness at $x=L-h, x=L$, and $y=-Y+h$. Finally, the solutions of (2)-(4) and (5), (6) are linked by the condition

$$
n(y=0)=\frac{1}{2 L} \int_{x=0}^{x=2 L} n(x) d x
$$

Numerical solution of the approximate model is of course straightforward. The qualitative solution form is the same as for the full model: highest NO concentration in the basal epidermis, with moderate NO levels in the epidermal peg, and low 

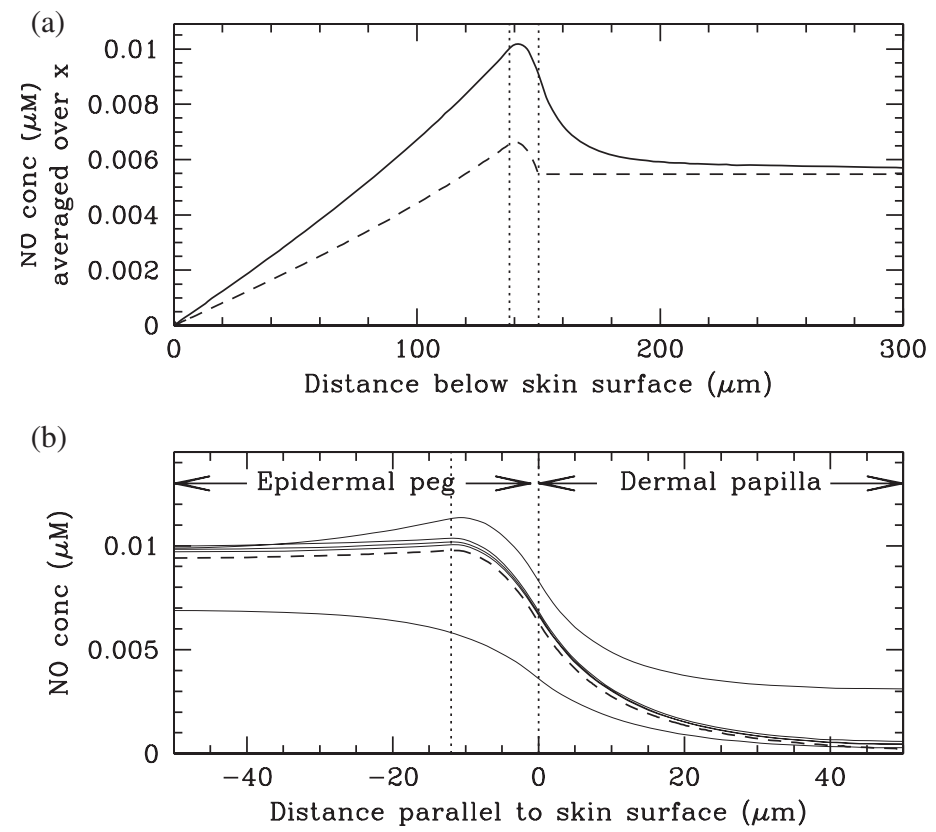

Figure 5. Comparisons of the solutions of the full model (1) ( - ) and approximate model (2)-(6) (- - - ) for NO distribution within a psoriatic plaque. In (a), we show the NO concentration averaged over $x$, and plotted as a function of $y$, the distance from the skin surface. The approximate solution is constant in the region $y<-Y$ because the model equations are ODEs for $n$ as a function of $x$ here. In (b), we show the NO concentration as a function of $x$ in the epidermal peg and dermal papillae. For the approximate model, this is just one solution, and for the full model, we plot the solution at 160, 200, 250, 300 , and $350 \mu \mathrm{m}$ below the skin surface; the solution decreases with increasing depth. Note that the full model solution decreases rapidly at depths above about $350 \mu \mathrm{m}$, because this is below the rete peg (see Fig. 2). In both (a) and (b), the dotted lines indicate the position of the basal epidermal layer. The figure shows that the solutions of the two models are qualitatively very similar, but that the approximate solution underestimates the NO concentration in the full solution. The parameter values are as in Fig. 3.

concentrations in the dermis. A quantitative comparison of the solutions of the full and approximate model (illustrated in Fig. 5) shows that the approximate model predicts NO concentrations that are too low, by a factor of as much as two. This is because the approximate model neglects the NO that diffuses into the dermal papillae from the basal epidermis above it. Similarly, within the upper region $\mathcal{U}$, there is diffusion of NO parallel to the skin surface, which redistributes the NO produced above the dermal papilla. These effects are appreciable because of the high diffusion coefficient of NO, and mean that the approximate model predicts NO levels that are too low throughout the upper layer $\mathcal{U}$ and the top of layer $\mathcal{L}$. Further down into the epidermal peg/dermal papilla, the full model solution becomes increasingly independent of distance below the skin surface, and thus approaches the solution of the approximate model. These comparisons imply that the approximate model underestimates both NO flux at the skin surface and the degree of 
erythema. However, we expect the qualitative variation in flux and erythema with model parameters to be the same in the two models.

\section{Solution of the Approximate Model}

We now derive a solution of the approximate model outlined earlier, beginning with the dermal layer. The basic objective of this work is to obtain formulae for the NO flux at the skin surface, given by $-D \partial n /\left.\partial y\right|_{y=0}$, and for the degree of erythema, defined within the approximate model as the average value of $1+g_{0} n / g_{1}$ within the dermal papillae (that is, the region $L<x<2 L$ ); these formulae are derived once the solution has been found.

4.1. Solution in the lower layer $\mathcal{L}$. Our approximate model divides the lower layer $\mathcal{L}$ into three regions $\mathcal{L}_{1}, \mathcal{L}_{2}$ and $\mathcal{L}_{3}$, corresponding, respectively, to the suprabasal and basal portions of the epidermal peg, and the dermal papilla. Equations (2) and (3), applying in $\mathcal{L}_{1}$ and $\mathcal{L}_{2}$, respectively, are solved easily, giving

$$
\begin{aligned}
n & =k_{1} \sinh (\sqrt{\lambda / D} x)+k_{2} \cosh (\sqrt{\lambda / D} x) \quad \text { in region } \mathcal{L}_{1} \\
\text { and } \quad n & =a / \lambda+k_{3} \sinh (\sqrt{\lambda / D} x)+k_{4} \cosh (\sqrt{\lambda / D} x) \quad \text { in region } \mathcal{L}_{2} .
\end{aligned}
$$

Here and in the following, the $k_{i}$ s are constants of integration; the condition $n_{x}=0$ at $x=0$ implies immediately that $k_{1}=0$. In $\mathcal{L}_{3}$, the solution satisfies (4), containing the nonlinearity that is key to NO dynamics. Nevertheless, exact solution is possible. Multiplying both sides of (4) by $n_{x}$ and integrating gives $n_{x}^{2}=$ $\alpha^{2} n^{2}+\frac{2}{3} \beta n^{3}$; the condition $n \rightarrow 0$ as $x \rightarrow \infty$ implies that the constant of integration is zero. This can then be solved by direct integration, giving

$$
n=\frac{6 \alpha^{2}}{\beta} \frac{k_{5} e^{\alpha x}}{\left(1-k_{5} e^{\alpha x}\right)^{2}} .
$$

The solutions (2)-(4) must satisfy continuity and smoothness at $x=L-h$ and $x=L$. In formulating these conditions it is convenient to write $\zeta=\sqrt{\lambda / D} L$, $\xi=\sqrt{\lambda / D}(L-h)$ and $\delta=k_{5} e^{\alpha L}$. Then, continuity and smoothness at $x=L-h$ imply

$$
\begin{aligned}
k_{2}-k_{4} & =(a / \lambda) \cosh \xi \\
k_{3} & =(a / \lambda) \sinh \xi .
\end{aligned}
$$

At $x=L$ the corresponding conditions are more complex because of the nonlinearity:

$$
a / \lambda+k_{3} \sinh \zeta+k_{4} \cosh \zeta=\frac{6 \alpha^{2}}{\beta} \frac{\delta}{(1-\delta)^{2}}
$$




$$
k_{3} \cosh \zeta+k_{4} \sinh \zeta=-\frac{6 \alpha^{2}}{\beta} \sqrt{1+v / \lambda} \frac{\delta(\delta+1)}{(\delta-1)^{3}} .
$$

Conditions (10)-(13) determine the constants of integration $k_{2}, k_{3}, k_{4}$ and $\delta$; recall that $\delta$ corresponds to $k_{5}$. It is straightforward to solve for $k_{2}, k_{3}$ and $k_{4}$ in terms of $\delta$, and eliminating $k_{3}$ and $k_{4}$ between (11), (12) and (13) gives

$$
\begin{aligned}
& \frac{a \beta}{6 \alpha^{2} \lambda}(\sinh \zeta-\sinh \xi)(\delta-1)^{3} \\
& =\{\sinh \zeta+\sqrt{1+v / \lambda} \cosh \zeta\} \delta^{2}+\{\sqrt{1+v / \lambda} \cosh \zeta-\sinh \zeta\} \delta .
\end{aligned}
$$

The algebraic complexity of the exact solutions of this cubic makes them of little practical use. However, it is easy to see that there is exactly one positive solution. Since the coefficients of $\delta$ and $\delta^{2}$ on the right-hand side of (14) are both positive, this right-hand side is zero at $\delta=0$, and is increasing on $\delta>0$. The left-hand side of (14) is also increasing on $\delta>0$, with a negative value at $\delta=0$; moreover, the left-hand side is larger than the right-hand side at large $\delta$. Hence there is at least one positive root; note that $\delta$ must be positive in order that the NO concentration implied by (9) is positive. Rewriting (14) as a cubic in $(\delta-1)$ and applying Descarte's rule of signs implies that this root is unique.

Before proceeding to the calculation of the solution in the upper layer $\mathcal{U}$, we must calculate $n_{0}$, the average NO concentration in regions $\mathcal{L}_{1}-\mathcal{L}_{3}$, which is required as an end condition in $\mathcal{U}$. This is given by

$$
n_{0}=\left(I_{1}+I_{2}+I_{3}\right) /(2 L)
$$

where

$$
\begin{aligned}
I_{1} & =\int_{x=0}^{L-h} k_{2} \cosh (\sqrt{\lambda / D} x) d x=k_{2} \sqrt{D / \lambda} \sinh \xi \\
I_{2} & =\int_{x=L-h}^{L} a / \lambda+k_{3} \sinh (\sqrt{\lambda / D} x)+k_{4} \cosh (\sqrt{\lambda / D} x) d x \\
& =a h / \lambda+\sqrt{D / \lambda}\left[k_{3} \cosh \zeta+k_{4} \sinh \zeta-k_{3} \cosh \xi-k_{4} \sinh \xi\right] \\
I_{3} & =\int_{x=L}^{2 L} \frac{6 \alpha^{2}}{\beta} \frac{k_{5} e^{\alpha x}}{\left(1-k_{5} e^{\alpha x}\right)^{2}} d x \\
& =\frac{6 \alpha}{\beta} \frac{\delta\left(e^{\alpha L}-1\right)}{(\delta-1)\left(\delta e^{\alpha L}-1\right)} .
\end{aligned}
$$

Summing these integrals and using (10), (11) and (13) gives

$$
n_{0}=\frac{1}{2 L}\left\{\frac{a h}{\lambda}+\frac{6 \alpha}{\beta} \frac{\delta}{\delta-1}\left[\frac{e^{\alpha L}-1}{\delta e^{\alpha L}-1}-\frac{\lambda+v}{\lambda} \frac{\delta+1}{(\delta-1)^{2}}\right]\right\} .
$$


At this point, we can also calculate our measure of erythema, the redness of the psoriatic plaque. This is one of the two key measures of our solution that we wish to calculate, the other being the NO flux at the skin surface. As discussed above, our definition of erythema is the average value of $g(n)$ in a dermal papilla. Within the context of our approximation $g(n) \approx 1+g_{0} n / g_{1}$, erythema is thus given by

$$
\begin{aligned}
\mathcal{E} & =1+\frac{g_{0}}{g_{1}} \frac{I_{3}}{L} \\
& =1+\frac{6 \sqrt{(\lambda+v) D}}{\nu L}\left[\frac{\delta\left(e^{\alpha L}-1\right)}{(\delta-1)\left(\delta e^{\alpha L}-1\right)}\right] .
\end{aligned}
$$

4.2. Solution in the upper layer $\mathcal{U}$. Having found the expression (16) for $n_{0}$, we can calculate the solution of our approximate problem in the upper layer $\mathcal{U}$. Here equations (5) and (6) apply in regions $\mathcal{U}_{1}$ and $\mathcal{U}_{2}$, respectively; the solution is now a function of $y(<0)$, the distance below the skin surface. Both equations are linear and can be solved immediately, to give

$$
\begin{aligned}
& \text { and } n=k_{6} \cosh (\sqrt{\lambda / D} y)+k_{7} \sinh (\sqrt{\lambda / D} y) \quad \text { in region } \mathcal{U}_{1} \\
& n=a /(2 \lambda)+k_{8} \cosh (\sqrt{\lambda / D} y) \\
& +k_{9} \sinh (\sqrt{\lambda / D} y) \quad \text { in region } \mathcal{U}_{2} .
\end{aligned}
$$

The constant $k_{6}=0$ in order that $n=0$ at $y=0$, and $k_{7}, k_{8}$ and $k_{9}$ are determined by the condition $n=n_{0}$ at $y=-Y$, and the requirements of continuity and smoothness at $y=-Y+h$; this is straightforward, and we omit the details.

All that we require from these solutions is the flux of $\mathrm{NO}$ at the skin surface $y=0$, which we denote by $\mathcal{F}$, and which is given by $-k_{7} \sqrt{\lambda D}$. The formula for $k_{7}$ implies

$$
\begin{aligned}
\mathcal{F} & =\frac{\sqrt{\lambda D}}{\sinh (\sqrt{\lambda / D} Y)}\left\{n_{0}+\frac{a}{2 \lambda}[\cosh (\sqrt{\lambda / D} h)-1]\right\} \\
= & \frac{\sqrt{\lambda D}}{L \sinh (\sqrt{\lambda / D} Y)}\left\{\mathcal{T}_{1}+\mathcal{T}_{2}\right\} \\
\text { where } \quad \mathcal{T}_{1}= & \frac{a L}{2 \lambda}[h / L+\cosh (\sqrt{\lambda / D} h)-1 \\
\text { and } \left.\quad-\sqrt{\frac{D}{L^{2} \lambda}} \frac{(\sinh \zeta-\sinh \xi)}{\cosh \zeta}\right] & \mathcal{T}_{2}=\frac{3 \alpha}{\beta}\left[\frac{\delta}{\delta-1} \frac{e^{\alpha L}-1}{\delta e^{\alpha L}-1}+\sqrt{1+v / \lambda} \frac{\delta}{(\delta-1)^{2}} \tanh \zeta\right] .
\end{aligned}
$$

Here we have simplified using (14) and (16). 
4.3. Variation in erythema and flux. Equations (17) and (20) are expressions for the degree of plaque erythema and the NO flux at the skin surface, within the context of our approximation (2)-(6) to the full mathematical model (1). Since NO is a potent vasodilator, one expects intuitively that there will be a correlation between how red a psoriatic plaque is, and the rate of NO release from its surface. However, experimental data indicates that although there is an overall positive correlation between NO release and erythema, NO release is highest in plaques with only a moderate degree of erythema; lower for paler plaques, but also lower for very red plaques. We will now use the formulae (17) and (20) to provide an explanation for these results.

Many of the parameters in our model for NO dynamics within a psoriatic plaque will be the same from one individual to the next. In particular, kinetic rate parameters and the NO diffusion coefficient are biochemical constants, and the spacing $2 L$ of dermal papillae is relatively constant between individuals. The epidermal thickness $Y$ will vary, but this has a simple, monotonic effect on NO release, and no effect on erythema (in our approximate system-the effect is also negligible in numerical solutions of the full model). Therefore we anticipate that variations between individuals will be due to differences in NO production in the basal epidermis, $a$, and in the degree of blood vessel dilation in response to NO, $g_{0}$ and $g_{1}$. In fact, more variation is to be expected in $g_{0}$, but the parameters enter our approximate model together, through $\beta$.

Therefore, we need to investigate how erythema $\mathcal{E}$ and NO flux $\mathcal{F}$ vary with the parameters $a$ and $\beta$. As a first step, we study the dependence of $\delta$ on these parameters; recall that $\delta$ is a positive constant of integration, determined uniquely by (14). The right-hand side of (14) is independent of $a$ and $\beta$, while the left-hand side is proportional to their product. Since the left-hand side is steeper than the right, as a function of $\delta$, when they cross, it follows that $\delta$ is a decreasing function of both $a$ and $\beta$.

From (17), it is immediately clear that $\mathcal{E}$ is a decreasing function of $\delta$. Thus, within our approximate model at least, the degree of plaque erythema increases with both $a$ and $\beta$. Turning to the NO release rate $\mathcal{F}$, we have separated formula (20) into two terms, labelled $\mathcal{T}_{1}$ and $\mathcal{T}_{2}$. The first of these is proportional to $a$, while the second is independent of $a$, implying that $\mathcal{F}$ increases with $a$. However, the dependence of $\mathcal{F}$ on $\beta$, which lies entirely in $\mathcal{T}_{2}$, requires more careful investigation, and is complicated by the lack of an explicit formula for $\delta . \mathcal{T}_{2}$ involves the two expressions

$$
\frac{\delta}{\beta(\delta-1)} \frac{e^{\alpha L}-1}{\delta e^{\alpha L}-1} \quad \text { and } \quad \frac{\delta}{\beta(\delta-1)^{2}}
$$

In the Appendix, we show that both of these expressions are decreasing functions of $\beta$, implying that $\mathcal{F}$ decreases with $\beta$. 


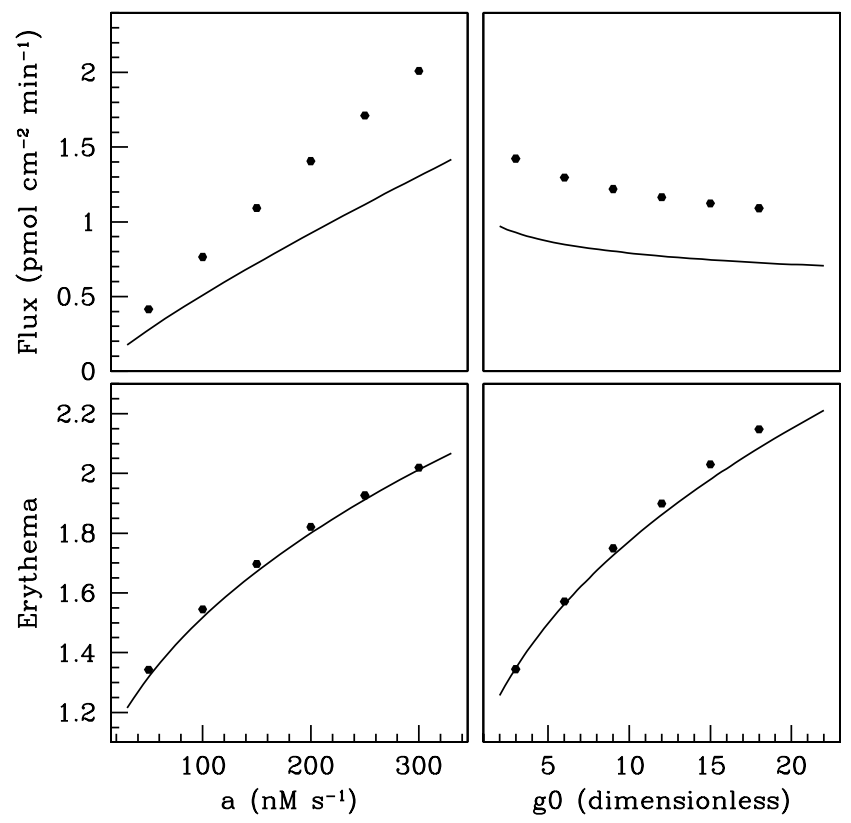

Figure 6. The variations in NO flux at the skin surface and in plaque erythema with parameters $a$ and $g_{0}$, in the full $(\bullet)$ and approximate (-) models. The parameter $g_{0}$ represents the dilation response of blood vessels to NO, while $a$ is the production rate of NO within the basal epidermis. The quantitative difference between the release rate and erythema predicted by the two models is expected from the difference in the solutions (see Fig. 5). Crucially, however, the qualitative variation in the solutions with these key parameters is very similar in the two models. The parameter values (other than $a$ or $g_{0}$ ) are as in Fig. 3.

4.4. Comparison with the full model. As we have discussed in Section 3, there are significant quantitative differences between the solution of the approximate model studied in this section, and the original full model. Our expectation, however, is that the qualitative solution trends that we have derived for the approximate model will be reflected in the full model, and this is borne out by numerical solutions. In Fig. 6, we show the variations of flux and erythema with the parameters $g_{0}$ and $a$ in the two models; the qualitative form of the variation is very similar in the two cases.

4.5. NO flux vs erythema. The variation in parameters $a$ and $g_{0}(\leftrightarrow \beta)$ between patients implies variations in NO flux and erythema. We have shown that the erythema is maximal when both $a$ and $g_{0}$ are greatest, that is, in patients with the largest NO production within the plaque, and the strongest vasodilation response to NO. In contrast, NO flux is maximal when $a$ is greatest, but $g_{0}$ is smallest. As observed experimentally, this corresponds to a moderate level of erythema. The predicted flux-erythema relationship is illustrated in Fig. 7. Here we have chosen many values of $a$ and $g_{0}$ randomly from normal distributions, and the figure shows 


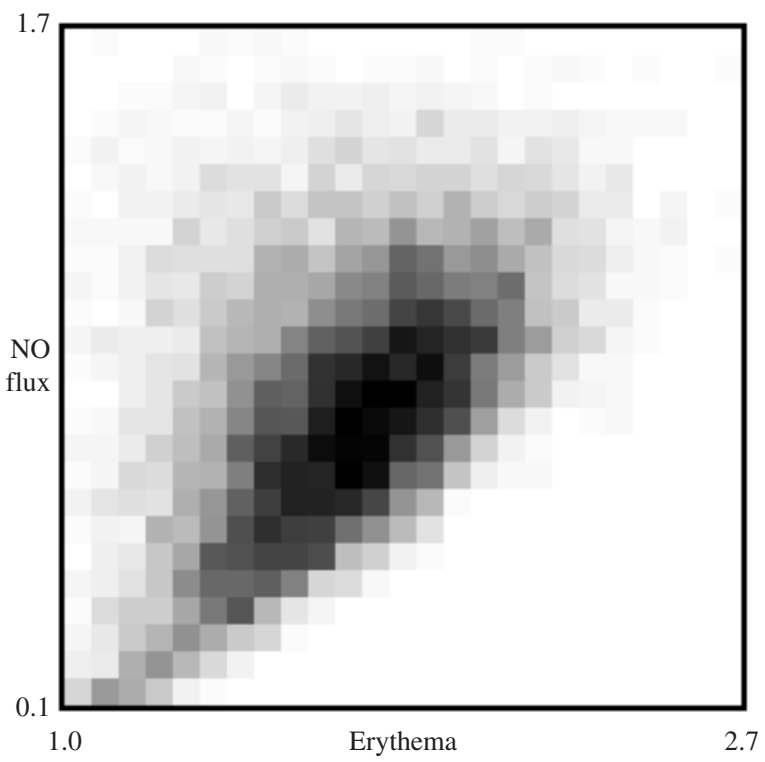

Figure 7. A prediction of the variation of NO flux at the skin surface with the erythema of a psoriatic plaque, determined using the approximate model. The figure shows that although there is positive correlation between NO flux and erythema, the maximum flux occurs in plaques with only moderate levels of erythema. In this figure, parameter values are as in Fig. 3, except for $a$ and $g_{0}$, which we expect to vary between patients. We chose pairs of values of $a$ and $g_{0}$ from independent normal distributions, with means of $170 \mathrm{nM} \mathrm{s}^{-1}$ and 9, respectively, based on experimental data (as discussed in Section 2). There is insufficient data to estimate the standard deviations, and we take these arbitrarily as $70 \mathrm{nM} \mathrm{s}^{-1}$ and 4 - thus the figure is intended to be illustrative, rather than a quantitative prediction. In the figure, 10000 pairs of parameter values are chosen, and the corresponding flux and erythema are calculated using (20) and (17). The flux-erythema plane illustrated is divided into a $25 \times 25$ grid, and each parameter pair gives a flux-erythema pair in one of these grid squares. (A small number fall outside the plot region and are ignored). We then shade each grid square using greyscale according to the number of parameter pairs giving values in this square (darker corresponds to more 'hits'). The units of NO flux are pmol cm-2 $\mathrm{min}^{-1}$.

a density plot of the corresponding predictions for $\mathrm{NO}$ release rate and plaque erythema.

\section{Discussion}

We have derived an analytical solution of our approximation (2)-(6) to the model (1) for NO dynamics within a psoriatic plaque. We have shown that this solution predicts that the degree of erythema and the rate of NO release at the skin surface will both increase as the rate of NO production in the basal epidermis increases. This is exactly as one would expect intuitively: higher production rates will increase NO levels throughout the plaque, causing a greater degree of blood 
vessel dilation, and higher release rates at the skin surface. This is clearly an important component of the variation between different psoriasis patients. However, the dilation response of blood vessels to NO will also differ from person to person, and the effects of this are less clear. A greater dilation response means that at a given NO concentration, there is faster removal by blood haemoglobin, leading to a balance between NO removal and the dilation caused by NO. Our analysis predicts that as the dilation response increases, the degree of erythema does in fact increase, while the NO flux at the skin surface goes down.

These predictions provide a possible explanation for the observation that the release rates of $\mathrm{NO}$ are highest in psoriatic plaques with moderate levels of erythema. Maximal NO release rates require high levels of NO production in the basal epidermis, and low dilation response of blood vessels to NO. Patients with stronger dilation responses will have lower NO release rates, but higher erythema, while for those with lower NO production in the basal epidermis of the plaque, the NO release rate and erythema will both be lower. In a study comparing various measures of psoriasis, Ormerod et al. (1997) did not find reduced NO release rates at high erythema; perhaps due to their particular patient group (of 12). Our model predicts that the phenomenon will be found in any sufficiently large study.

Comparison between the model predictions (Fig. 7) and experimental data (Fig. 1) shows one clear qualitative difference, with a few measurements of particularly high NO release rates that are not reflected by the model. We hypothesize that these 'outliers' correspond to additional production of NO in suprabasal, as well as basal, epidermis; suprabasal keratinocytes have been found to express iNOS mRNA in a minority of psoriatic plaques (Bruch-Gerharz et al., 1996). Aside from this difference, there is good semi-quantitative comparison between the model and experiments. Differences can be explained by variations in plaque structure not reflected in the simulations, and by the approximate nature of the relationship between model calculations of erythema and experimental scores.

NO levels are very high in psoriatic plaques: release rates are an order of magnitude greater than in nonlesional skin, and two orders of magnitude greater than healthy skin. This raises immediate and currently unanswered questions about the possible role for NO in onset of psoriasis; our modelling does not address this at all, considering only NO dynamics within a pre-existing plaque. The potential for NO having a causal effect in plaque formation arises from the many aspects of skin biology that are regulated by NO, including epidermal cell division and differentiation (Krischel et al., 1998), endothelial cell growth and migration (Ziche et al., 1994), and extracellular matrix turnover (Okamoto et al., 1997; Maeda et al., 1998). In a separate study (Savill et al., 2002), we have used mathematical modelling to investigate this last effect, suggesting that the NO-dependence of matrix degradation may help to explain the elongated rete pegs observed in psoriatic plaques.

The long-term motivation for mathematical modelling and experimental work on NO in psoriasis is the possibility of new anti-psoriasis therapies, based on NO activity. This paper contributes to the basic understanding of NO dynamics in pso- 
riatic plaques, which will underlie any such therapy. More specifically, our work shows that the nonlinearity in the uptake of NO by blood vessels is fundamental to NO dynamics, and provides a clear explanation for experimental results on NO release rates from psoriatic plaques.

\section{APPENDIX}

In this Appendix, we show that the two expressions in (21) are decreasing functions of $\beta$. The complication in this is the dependence on $\delta$, for which we have no exact formula, only equation (14). It is convenient to work with the reciprocals of the expressions in (21); thus we aim to show that $\phi \equiv \beta(\delta-1)^{2} / \delta$ and $\psi \equiv \beta(\delta-1)\left(\delta e^{\alpha L}-1\right) / \delta$ are increasing functions of $\beta$. We consider $\phi$ first.

Rewriting (14) gives

$$
\begin{aligned}
{\left[\frac{a}{6 \alpha^{2} \lambda}(\sinh \zeta-\sinh \xi)\right] \phi=} & {[\sinh \zeta+\sqrt{1+v / \lambda} \cosh \zeta] } \\
& +[2 \sqrt{1+v / \lambda} \cosh \zeta] \frac{1}{\delta-1}
\end{aligned}
$$

Simple rearrangement of the definition of $\phi$ implies that $\beta(\delta-1)^{2}-\phi(\delta-1)-\phi=$ 0 ,

$$
\Rightarrow(\delta-1)=\frac{\phi+\sqrt{\phi^{2}+4 \phi \beta}}{2 \beta} .
$$

Here the positive root of the quadratic is required since the positive root of (14) is necessarily greater than 1 . Substituting this formula for $\delta-1$ into (22) gives

$$
\begin{aligned}
{\left[\frac{a}{6 \alpha^{2} \lambda}(\sinh \zeta-\sinh \xi)\right] \phi=} & {[\sinh \zeta+\sqrt{1+v / \lambda} \cosh \zeta] } \\
& +[4 \sqrt{1+v / \lambda} \cosh \zeta] \frac{\beta}{\phi+\sqrt{\phi^{2}+4 \beta \phi}}
\end{aligned}
$$

This equation gives the relationship between $\phi$ and $\beta$. Considering $\phi$ as a variable in this equation, the left-hand side has a linearly increasing dependence, while the right-hand side is a decreasing function of $\phi$. Therefore, in order to show that $\phi$ increases with $\beta$, it is sufficient to show that $\beta /\left(\phi+\sqrt{\phi^{2}+4 \beta \phi}\right)$ is an increasing function of $\beta$, for each fixed $\phi>0$. This follows easily from direct differentiation:

$$
\begin{aligned}
& \left.\frac{\partial}{\partial \beta}\left[\frac{\beta}{\phi+\sqrt{\phi^{2}+4 \beta \phi}}\right]\right|_{\phi \text { const }} \\
& =\frac{\phi}{\sqrt{\phi^{2}+4 \beta \phi}\left(\phi+\sqrt{\phi^{2}+4 \beta \phi}\right)^{2}}\left[\sqrt{\phi^{2}+4 \beta \phi}+\phi+2 \beta\right]>0 .
\end{aligned}
$$


Having established that $\phi$ increases with $\beta$, it is straightforward to show that $\psi$ does also. Rewriting the definition of $\psi$ gives

$$
\psi=\phi \cdot\left[\frac{e^{\alpha L} \delta-1}{\delta-1}\right],
$$

and direct differentiation shows that the term inside square brackets is a decreasing function of $\delta$, and thus an increasing function of $\beta$.

\section{ACKNOWLEDGements}

We thank Patrick Wright for very helpful discussions. JAS was supported in part by an Advanced Research Fellowship from EPSRC. NJS and JAS were supported in part by Research Development Grant 107 ('Centre for Theoretical Modelling in Medicine') from SHEFC.

\section{REFERENCES}

Auer, T., M. Bacharach-Buhles, S. el Gammal, M. Stücker, B. Panz, C. Popp, K. Hoffmann, M. Happe and P. Altmeyer (1994). The hyperperfusion of the psoriatic plaque correlates histologically with dilation of vessels. Acta Derm. Venereol. S186, $30-32$.

Bruch-Gerharz, D., K. Fehsel, C. Suschek, G. Michel, T. Ruzicka and V. Kolb-Bachofen (1996). A proinflammatory activity of interleukin 8 in human skin: expression of the inducible nitric oxide synthase in psoriatic lesions and cultured keratinocytes. J. Exp. Med. 184, 2007-2012.

Ignarro, L. J., R. E. Byrns, G. M. Buga and K. S. Wood (1987). Endothelium derived relaxing factor from pulmonary artery and vein possesses the pharmacologic and chemical properties identical to those of nitric oxide radical. Circ. Res. 61, 866-879.

Klemp, P. and B. Staberg (1983). Cutaneous blood flow in psoriasis. J. Invest. Dermatol. 81, 503-506.

Kolb-Bachofen, V., K. Fehsel, G. Michel and T. Ruzicka (1994). Epidermal keratinocyte expression of inducible nitric oxide synthase in skin lesions of psoriasis vulgaris. Lancet 344, 139.

Krischel, V., D. Bruch-Gerharz, C. Suschek, K. D. Kroncke, T. Ruzicka and V. KolbBachofen (1998). Biphasic effect of exogenous nitric oxide on proliferation and differentiation in skin derived keratinocytes but not fibroblasts. J. Invest. Dermatol. 111, 286-291.

Krogstad, A. L., G. Swanbeck and B. G. Wallin (1995). Axon reflex mediated vasodilation in the psoriatic plaque? J. Invest. Dermatol. 104, 872-876.

Ku, D. D. (1996). Nitric oxide and nitric oxide donor induced relaxation. Methods Enzymol. 269, 107-119. 
Lancaster, J. R. (1994). Simulation of the diffusion and reaction of endogenously produced nitric oxide. Proc. Natl. Acad. Sci. USA 91, 8137-8141.

Liao, J. C., T. W. Hein, M. W. Vaughn, K. T. Huang and L. Kou (1999). Intravascular flow decreases erythrocyte consumption of nitric oxide. Proc. Natl. Acad. Sci. USA 96, 8757-8761.

Maeda, H., T. Okamoto and T. Akaike (1998). Human matrix metalloprotease activation by insults of bacterial infection involving proteases and free radicals. Biol. Chem. 379, 193-200.

Malinski, T., Z. Taha, S. Grunfeld, S. Patton, M. Kapturczak and P. Tomboulian (1993). Diffusion of nitric oxide in the aorta wall monitored in situ by porphyrinic microsensors. Biochem. Biophys. Res. Commun. 193, 1076-1082.

Moncada, S. (1999). Nitric oxide: discovery and impact on clinical medicine. J. R. Soc. Med. 92, 164-169.

Okamoto, T., T. Akaike, T. Nagano, S. Miyajima, M. Suga, M. Ando, K. Ichimori and H. Maeda (1997). Activation of human neutrophil procollagenase by nitrogen dioxide and peroxynitrite: a novel mechanism for procollagenase activation involving nitric oxide. Arch. Biochem. Biophys. 342, 261-274.

Orem, A., R. Aliyazicioglu, E. Kiran, B. Vanizor, G. Cimnocodeit and O. Deger (1997). The relationship between nitric oxide production and activity of the disease in patients with psoriasis. Arch. Dermatol. 133, 1606-1607.

Ormerod, A. D., C. M. Dwyer, R. Weller, D. H. Cox and R. Price (1997). A comparison of subjective and objective measures of reduction of psoriasis with the use of ultrasound, reflectance colorimetry, computerized video image analysis and nitric oxide production. J. Am. Acad. Dermatol. 37, 51-57.

Ormerod, A. D., R. Weller, P. Copeland, N. Benjamin, S. H. Ralston, P. Grabowksi and R. Herriot (1998). Detection of nitric oxide and nitric oxide synthases in psoriasis. Arch. Dermatol. Res. 290, 3-8.

Savill, N. J., R. Weller and J. A. Sherratt (2002). Mathematical modelling of nitric oxide regulation of rete peg formation in psoriasis. J. Theor. Biol. 214, 1-16.

Thibodeau, G. A. and K. T. Patton (1997). The Human Body in Health and Disease, St Louis: Mosby Year Book Inc.

Weller, R. and A. Ormerod (1997). Increased expression of nitric oxide synthase. Br. J. Dermatol. 136, 132-148.

Weller, R., A. Ormerod and N. Benjamin (1996). Nitric oxide generation measured directly from psoriatic plaques by chemiluminescence. Br. J. Dermatol. 134, 569.

Ziche, M., L. Morbidelli, E. Masini, S. Amerini, R. H. J. Grange, C. A. Maggi, P. Geppetti and F. Ledda (1994). Nitric oxide mediates angiogenesis in vivo and endothelial cell growth and migration in vitro promoted by substance P. J. Clin. Invest. 94, 2036-2044. 